\title{
Removal of mechanical ventilation during veno- venous ECMO may improve outcome of patients with acute respiratory failure due to adult community-acquired pneumonia
}

\section{Lu-Lu Li}

Zhejiang University

Cong-Ying Song

Zhejiang University

Mei Shao

Zhejiang University

An-Dong Shang

Zhejiang University

Lin-Lin Meng

Zhejiang University

Yuan-Qiang Lu ( $\nabla$ luyuanqiang@zju.edu.cn )

Zhejiang University

\section{Research Article}

Keywords: ARF, Mechanical ventilation, ECMO, Prognosis

Posted Date: October 12th, 2021

DOI: https://doi.org/10.21203/rs.3.rs-798903/v2

License: (c) (i) This work is licensed under a Creative Commons Attribution 4.0 International License.

Read Full License 


\section{Abstract \\ Background}

As a life-saving therapy for patients with acute respiratory failure (ARF)Mechanical ventilation has catalyzed the development of modern emergency medicine and intensive care units.Another way to support respiratory or cardiac functions is extracorporeal membrane oxygenation (ECMO). Based on previous studies, the increased pre-ECMO time of mechanical ventilation is a significant independent predictor of the poorer outcome. Removal or maintaining of mechanical ventilation during ECMO is still debatable.

\section{Methods}

We analyzed the clinical data of 23 patients veno-venous ECMO therapy with acute respiratory failure due to adult community-acquired pneumonia.They were divided into two groups: group A (removed of mechanical ventilation, $n=10$ ) and group $B$ (maintaining of mechanical ventilation, $n=13$ ). Demographic data, including gender, age, smoking habits were collected. General characteristics and Clinical characteristics of patients were also recorded, in order to discuss whether the retention or removal of trachea cannula and continued mechanical ventilation during ECMO can affect patients' prognosis.

\section{Results}

After analysis, patients in the Group B were older than the Group A (61.0 y [54.5-67.5] vs 39.0 y [24.061.8], $P=0.021)$. The median APACHE $\otimes$ score of 23 patients before ECMO therapy was 25.0 (IQR, 21.028.0), and the Group A had a lower initial APACHE $₫$ score than the Group B (21.5 [20.8-24.3] vs 28.0 [24.0-29.0], $P=0.005$ ). The group $A$ with a survival rate of $80 \%$, and the group $B$ presenting a survival rate of $23.1 \%$. The difference in the survival rate between the two groups was statistically significant $(P=$ 0.012).No differences in other items were found between the two groups.

\section{Conclusions}

The final results showed that the removing of mechanical ventilation during ECMO can improve the survival rate and prognosis in patients with ARF.

\section{Introduction}

Mechanical ventilation as a life-saving therapy for patients with acute respiratory failure (ARF) has catalyzed the development of modern emergency medicine and intensive care units (1). However, mechanical ventilator is a double-edged sword that not only provide effective breathing for the patients, but also associates with a host of complications to exacerbate lung injury $(1,2)$. Another means of 
supporting respiratory or cardiac functions is extracorporeal membrane oxygenation (ECMO). Although the use of ECMO in cardiopulmonary disease is evolving rapidly, many questions about the optimal patient populations and the timing for initiation of ECMO remain to be solved (3). Based on previous studies, the increased pre-ECMO time of mechanical ventilation is a significant independent predictor of the poorer outcome (4). Especially for patients with severe acute respiratory distress syndrome, the preECMO mechanical ventilation lasting at least 7 days has been associated with higher mortality $(5,6)$. Removal or maintaining of mechanical ventilation during ECMO is still debatable. Thus, we analyzed the clinical data of 23 patients veno-venous ECMO therapy with acute respiratory failure due to adult community-acquired pneumonia.

\section{Data And Methods}

\subsection{Selection of participants}

There were all 103 patients who received extracorporeal support in the emergency care unit at the First Affiliated Hospital, School of Medicine, Zhejiang University between November 2014 and September 2019. Patients who met any of the following criteria were excluded: (1) cardiac failure resulting in venoarterial ECMO, (2) ECMO provided for other indications than ARF,(3) pregnancy,(4) longterm chronic respiratory insufficiency treated with oxygen therapy or noninvasive ventilation.Following the inclusion and exclusion criteria, 80 parents were excluded and 23 parents were included, and the specific screening process was shown in Fig. 1. All included cases were severe pneumonia, including 12 cases of viral pneumonia and 11 cases of undefined pathogen infection (Fig. 1).

\subsection{Data collection}

Demographic data, including gender, age, smoking habits were collected. The clinical parameters were also recorded, such as ECMO duration, and initial acute physiology and chronic health evaluation (APACHE) \score. Also, we collected many basic characteristics: The patients' heart rate(HR), mean arterial pressure (MAP), temperature $(\mathrm{T})$ were continuous recorded by the monitors; ECMO parameters: rotational speed Flow, sweep gas flow, fraction of inspired oxygen(FiO2);Artery blood gas analyzer was used for recording partial pressure of arterial carbon dioxide ( $\mathrm{PaCO} 2)$, partial pressure of arterial oxygen $(\mathrm{PaO} 2)$, lactic acid ( $\mathrm{Lac})$ and potential of hydrogen $(\mathrm{pH})$ value of patients. These indicators were used to monitor the hemodynamics, the patient's internal environment and whether the ECMO machine was working well in two groups, in order to look for the influence on the prognosis.

\subsection{ECMO operation conditions and deployment}

We selected patients with ARF who with mechanical ventilation less than 7 days by one of three criteria a ratio of partial pressure of arterial oxygen $\left(\mathrm{PaO}_{2}\right)$ to the fraction of inspired oxygen $\left(\mathrm{FiO}_{2}\right)$ of less than $50 \mathrm{mmHg}$ for more than 3 hours, a $\mathrm{PaO}_{2}: \mathrm{FiO}_{2}$ of less than $80 \mathrm{mmHg}$ for more than 6 hours, or an arterial blood potential of hydrogen $(\mathrm{pH})$ of less than 7.25 with a partial pressure of arterial carbon dioxide 
$\left(\mathrm{PaCO}_{2}\right)$ of at least $60 \mathrm{mmHg}$ for more than 6 hours-to receive immediate venovenous ECMO. ARF was defined as clinical evidence of respiratory distress (tachypnea, dyspnea) and hypoxemia at supplementary oxygen rates greater than or equal to $10 \mathrm{~L} /$ min (high-flow oxygen or non-invasive ventilation) or mechanical ventilation.

ECMO deployment: all patients were using silica membrane lungs (manufacturer: MAQUET; model: Rotaflow, made in Germany) in veno-venous mode. Percutaneous cannulation was performed using the Seldinger technique. The right internal jugular vein and right femoral vein were used for venous cannula. Under deep sedation and after the successful deployment of ECMO, the perfusion flow should be improved; the membrane oxygenation concentration was set at $70 \%-80 \%$, and the ratio of gas flow and blood flow was $0.5-0.8: 1$. Then parameters were adjusted according to the heart rate (HR), blood pressure, central venous pressure (CVP), and blood gas. At the same time, the mechanical ventilation was adjusted to low voltage and low frequency mode with $20-24 \mathrm{cmH}_{2} \mathrm{O}$ peak pressure, $10 \mathrm{mmHg}$ positive end-expiratory pressure (PEEP), 5-10 times/min frequency, and $21 \%-40 \% \mathrm{FiO}_{2}$. Unfractionated heparin (UFH) was used in all patients, with a bolus at the dose of 50-100 $\mathrm{U} / \mathrm{kg}$ before cannulation. Then a continuous intravenous infusion of UFH was increased at a rate of 2-to-20 U/ kg/h, with a target activated clotting time (ACT) level of 180-to-200 seconds or an activated partial thromboplastin time (APTT) of 60to-80 seconds. Point-of-care monitoring was conducted for ACT or APTT(every 4 hours), and the APTT, thrombin time, prothrombin time, fibrinogen, D-dimer, and platelet count were monitored every 24 hours. Early mechanical ventilation removal can be considered for ECMO patients who meet the following conditions: the primary disease has been controlled to a certain extent;Be conscious;Airway protection was strong, and there was no obvious airway secretions or airway inflammation in bronchoscopy.Hemodynamic stability, no serious arrhythmia; In the auxiliary ventilation mode, peak pressure $\leq 20 \mathrm{cmH} 2 \mathrm{O}, \mathrm{PEEP} \leq 10 \sim 12 \mathrm{cmH} 2 \mathrm{O}, \mathrm{FiO} 2 \leq 0.5$. High risk or suspected possibility of Ventilatorassociated pneumonia (VAP); The presence or high risk of barotrauma;ECMO operation was normal, coagulation and fibrinolysis indexes were stable, there were no obvious signs of infection at the vascular puncture site, and there were no inclusions of serious complications. according to whether patients were removed of mechanical ventilation or not during ECMO, 23 cases were divided into two groups: group $A$ (removed of mechanical ventilation, $n=10$ ) and group $B$ ( maintaining of mechanical ventilation, $n=13$ ). This study was approved by the Ethical Committee of First Affiliated Hospital, School of Medicine, Zhejiang University. We obtained the consent of all patients or their relatives.

\subsection{Statistical analysis}

Continuous variables were described as medians with interquartile ranges (IQR). Categorical variables were shown as numbers and percentages. Mann-Whitney U-test was performed for evaluating continuous data and Chi-square test for categorical variables. Survival analysis was performed by the Kaplan-Meier method and evaluated by the log-rank test. The level of statistical significance was set at $P<0.05$. All analyses were conducted with SPSS software (version 25.0, SPSS Inc., IBM, Chicago, IL, USA)

\section{Results}




\subsection{General characteristics of patients}

Of 23 participants we recruited, there were 13 males and 10 females, while, no differences in sex ratio were found between the two groups $(P=0.768)$. Patients in the Group B were older than the Group A (61.0 $y$ [54.5-67.5] vs 39.0 y [24.0-61.8], $P=0.021)$. The rate of smoker was higher in the group $B$, but there was no significant difference between the two groups $(P=0.673)$. The median duration of ECMO of 23 patients was 12 days (range 5-47 days), and its difference between the two groups was not statistically significant $(P=0.483)$. APACHE $\otimes$ was a classification system to measure the severity of disease for adult patients admitted to Intensive Care Unit (ICU). The median APACHE $\triangle$ score of 23 patients before ECMO therapy was 25.0 (IQR, 21.0-28.0), and the Group A had a lower initial APACHE $\triangle$ score than the Group B (21.5 [20.8-24.3] vs 28.0 [24.0-29.0], $P=0.005$ )(Table 1 ).

Table 1

General information of ECMO patients

\begin{tabular}{|lllll|}
\hline Parameters & $\begin{array}{l}\text { group A } \\
(\mathbf{n = 1 0 )}\end{array}$ & $\begin{array}{l}\text { group B } \\
(\mathbf{n = 1 3})\end{array}$ & Zor $x^{2}$ & Pvalue \\
\hline Male, $\mathbf{n}(\%)$ & $6(60.0)$ & $7(53.8)$ & 0.087 & 0.768 \\
\hline Age, $\mathbf{y}$ & $39.0(24.0-61.8)$ & $61.0(54.5-67.5)$ & -2.266 & 0.021 \\
\hline Smokers, $\mathbf{n}(\%)$ & $3(30.0)$ & $5(38.5)$ & 0.178 & 0.673 \\
\hline ECMO duration, $\mathbf{d}$ & $11.0(7.5-17.3)$ & $17.0(7.0-19.0)$ & -0.748 & 0.483 \\
\hline Initial APACHE score & $21.5(20.8-24.3)$ & $28.0(24.0-29.0)$ & -2.725 & 0.005 \\
\hline Data are presented as medians (interquartile ranges, IQR) or N (\%). & \\
\hline
\end{tabular}

\subsection{Clinical characteristics of patients}

Basic characteristics, ECMO parameters, and blood gas parameters during ECMO and 24 hours after ECMO are shown in Fig. 2. No significant difference between the two groups was found in all parameters (all $P>0.05$ ).

Table 2 shows the basic characteristics, blood gas, and ECMO parameters of the group A 24 hours before and after removing of mechanical ventilation. The significant increases in ECMO parameters (rotational speed, flow of ECMO, sweep gas flow, and $\mathrm{FiO}_{2}$ ) were noted 24 hours after removing of mechanical ventilation in the group $\mathrm{A}$, but no difference was found $(P>0.05)$. Compared to the group $\mathrm{A}$, the group $\mathrm{B}$ had a higher Lac at all three times, but no difference was found $(P>0.05)$. 
Table 2

Basic characteristics, ECMO parameters and blood gas parameters of the group A 24 hours before and after removing of mechanical ventilation

\begin{tabular}{|c|c|c|c|}
\hline & Before & After & $P$ value \\
\hline \multicolumn{4}{|l|}{ Basic characteristics } \\
\hline HR, beats/min & 89.6 (77.5-105.4) & $86.7(77.0-101.6)$ & 0.853 \\
\hline $\mathrm{T},{ }^{\circ} \mathrm{C}$ & $37.0(36.9-37.4)$ & $37.0(36.8-37.2)$ & 0.796 \\
\hline MAP, mmHg & $87.8(81.5-95.8)$ & $93.3(84.2-104.2)$ & 0.280 \\
\hline \multicolumn{4}{|l|}{ ECMO parameters } \\
\hline Rotational speed, rpm & $3207.8(3042.2-3535.9)$ & $3348.6(3207.8-3546.9)$ & 0.280 \\
\hline Flow, L/min & $4.1(4.0-4.3)$ & $4.2(4.1-4.6)$ & 0.315 \\
\hline Sweep gas flow, L/min & $5.1(4.0-6.5)$ & $6.2(5.3-6.4)$ & 0.353 \\
\hline FiO2 & $74.9(67.5-90.3)$ & $82.7(59.0-100.0)$ & 0.739 \\
\hline \multicolumn{4}{|l|}{ Blood gas parameters } \\
\hline $\mathrm{pH}$ & $7.48(7.43-7.50)$ & $7.49(7.43-7.51)$ & 0.529 \\
\hline $\mathrm{PaO} 2$ & $96.8(70.3-124.8)$ & $95.5(77.0-161.4)$ & 0.796 \\
\hline $\mathrm{PaCO} 2$ & $33.7(31.9-34.9)$ & $32.9(24.7-38.0)$ & 0.684 \\
\hline $\mathrm{Lac}, \mathrm{mg} / \mathrm{dL}$ & $1.8(1.1-2.4)$ & $1.9(1.1-2.7)$ & 0.796 \\
\hline
\end{tabular}

\subsection{Survival analysis}

Figure 3 shows the survival curve of all patients, the group A with a survival rate of $80 \%$ ( 8 out of 10 patients were survived), and the group B presenting a survival rate of $23.1 \%$ (3 out of 13 patients were survived). The difference in the survival rate between the two groups was statistically significant $(P=$ 0.012).

\section{Discussion}

The ARF is characterized by lung injury caused by either indirect or direct insults, which could be worsened by the way mechanical ventilation is applied (7). ECMO has been used in clinical medicine for over 40 years but remains controversial. As a result, it is considered a rescue therapy when conventional management fails (8). With ECMO, it is possible to rest the lungs by using lower tidal volumes, lower airway pressures, and lower $\mathrm{FiO}_{2}$, thereby decreasing the iatrogenic consequences of mechanical ventilation (9). Initiation of ECMO allows reductions in the tidal volume size, PEEP, and plateau pressure 
levels, as well as $\mathrm{FiO}_{2}(9-11)$, which all may help to improve outcome via prevention of additional lung injury $(12,13)$.

This study is to discuss whether the retention or removal of trachea cannula and continued mechanical ventilation during ECMO can affect patients' prognosis. It is generally accepted that the retention of trachea cannula and continued use of mechanical ventilation in the low voltage and low frequency mode during ECMO can improve alveolar oxygen partial pressure and decrease pulmonary vascular resistance, and the higher PEEP can prevent atelectasis.

Technically, the ECMO should be able to provide enough oxygen patients need. According to previous clinical practices and research, the removal of trachea cannula during ECMO can help patients with ARF to gain comfort, to reduce the stress level, use of sedatives and interference to circulation, and to promote cough and sputum. However, the removal of mechanical ventilation can also bring some disadvantages: for patients with poor lung compliance, it may lead to large atelectasis; for patients with ARF infected by the high aggressive pathogenic microorganism, it can result in partial alveolar collapse and may spread infection in lungs; due to the reduced oxygen supply by mechanical ventilation, ECMO needs to be enhanced, thereby increasing the incidence and intensity of adverse reactions, such as blood damage caused by excessive flow.

All in all, 23 patients with ECMO had no neuromuscular diseases and no significant abnormality in their respiratory center and respiratory drive. After patients with ARF were extubated tracheal cannula and stopped mechanical ventilation for 24 hours, ECMO-supported parameters slightly improved, while patients' HR, T, MAP, and Lac improved better than those before removing of mechanical ventilation. Thus, tracheal extubation during ECMO seems to greatly increase the tissue oxygenation level of patients, which may be related to the improvement of balance between oxygen supply and consumption. The final results also showed that the removing of mechanical ventilation during ECMO seemed to improve the survival rate and prognosis in patients with ARF. Of course, patients in the two groups have a statistically significant difference in terms of age and APACHE $\triangle$ score, which is consistent with the clinical practice. Young and mild patients are more likely to remove tracheal intubation.

Given the small sample capacity, this study is unrealistic and unscientific to get definite results. It is expected to draw clear conclusions from subsequent multicentric and large-scale clinical research and to make in-depth discussions on various mechanisms involved, in order to use ECMO more scientifically and save more patients.

\section{Conclusions}

We found that the elimination of mechanical ventilation during ECMO can improve the survival and prognosis of ARF patients,tracheal extubation during ECMO seems to greatly increase the tissue oxygenation level of patients. Of course,there were statistically significant differences in age and APACHE 
$\nabla$ score between the two groups, which was consistent with clinical practice. Young and mild patients are more likely to pull out endotracheal intubation.

\section{Abbreviations}

ECMO: extracorporeal membrane oxygenation; ARF: acute respiratory failure; APACHEA: acute physiology and chronic health evaluation;HR: heart rate; T: temperature;MAP: mean arterial pressure; $\mathrm{pH}$ : potential of hydrogen; $\mathrm{PaO2}$ : partial pressure of arterial oxygen; $\mathrm{PaCO}$ : partial pressure of arterial carbon dioxide; Lac: lactic acid; FiO2: fraction of inspired oxygen; CVP:central venous pressure;PEEP:positive endexpiratory pressure;UFH:unfractionated heparin;APTT:activated partial thromboplastin time;ACT:activated clotting time;IQR:interquartile ranges; ICU:Intensive Care Unit;VAP:Ventilator-associated pneumonia.

\section{Declarations}

\section{Acknowledgements}

We would like to thank for Tong-Li, Ph.D., providing the help on statistical analyses.

\section{Authors contributions}

YQL conceived and designed the experiments. LLL andMScollected and analyzed the data.LLL and CYS contributed to the writing of the manuscript.ADS and LLM edited figures and charts. YQL revised the manuscript.All authors read and approved the final manusscript.

\section{Funding}

This work was supported by the Key Research and Development Program of Zhejiang Province (No. 2019C03076) and the Foundation of Key Discipline Construction of Zhejiang Province for Traditional Chinese Medicine (No. 2017-XK-A36).

\section{Availability of data and materials}

The datasets used and/or analyzed during the current study are available from the corresponding author on reasonable request.

\section{Ethics approval and consent to participate}

This study was approved by the Ethical Committee of the First Affiliated Hospital, School of Medicine, Zhejiang University (code number IIT20210464A). Written consent was obtained from each patient or his/her authorized representatives following a full explanation of the study.

\section{Consent for publication}

Not applicable. 


\section{Competing interests}

The authors declare that they have no competing interests.

\section{Author information}

${ }^{1}$ Department of Emergency Medicine and Zhejiang Provincial Key Laboratory for Diagnosis and Treatment of Aging and Physic-chemical Injury Diseases, The First Affiliated Hospital, School of Medicine, Zhejiang University, 79 Qingchun Road, Hangzhou, 310003 Zhejiang, People's Republic of China. ${ }^{2}$ State Key Laboratory for Diagnosis and Treatment of Infectious Diseases, The First Affiliated Hospital, School of Medicine, Zhejiang University, 79 Qingchun Road, Hangzhou, 310003 Zhejiang, People's Republic of China

\section{References}

1. Slutsky AS. History of Mechanical Ventilation. From Vesalius to Ventilator-induced Lung Injury. American journal of respiratory and critical care medicine. 2015;191(10): 1106-1115.

2. Walkey AJ, Summer R, Ho V, Alkana P. Acute respiratory distress syndrome: epidemiology and management approaches. Clinical Epidemiology. 2012;4: 159-169.

3. Abrams D, Combes A, Brodie D. Extracorporeal Membrane Oxygenation in Cardiopulmonary Disease in Adults. J Am Coll Cardiol. 2014;63(25): 2769-2778.

4. Kolla S, Awad SS, Rich PB, Schreiner RJ, Hirschl RB, Bartlett RH. Extracorporeal life support for 100 adult patients with severe respiratory failure. Annals of surgery. 1997;226(4): 544 - 64; discussion 65-66.

5. Schmidt M, Bailey M, Sheldrake J, Hodgson C, Aubron C, Rycus PT, et al. Predicting survival after extracorporeal membrane oxygenation for severe acute respiratory failure. The Respiratory Extracorporeal Membrane Oxygenation Survival Prediction (RESP) score. American journal of respiratory and critical care medicine. 2014;189(11): 1374-1382.

6. Schmidt M, Zogheib E, Roze H, Repesse X, Lebreton G, Luyt CE, et al. The PRESERVE mortality risk score and analysis of long-term outcomes after extracorporeal membrane oxygenation for severe acute respiratory distress syndrome. Intensive care medicine. 2013;39(10): 1704-1713.

7. Ware LB, Matthay MA (2000) The acute respiratory distress syndrome. N Engl J Med 342: 13341337

8. Schmidt M, Brechot N, Combes A. Ten situations in which ECMO is unlikely to be successful. Intensive care medicine. 2016;42(5): 750-752.

9. Peek GJ, Mugford M, Tiruvoipati R, Wilson A, Allen E, Thalanany MM, Hibbert CL, Truesdale A, Clemens F, Cooper N, Firmin RK, Elbourne D, CESAR trial collaboration (2009) Efficacy and economic assessment of conventional ventilatory support versus extracorporeal membrane oxygenation for severe adult respiratory failure (CESAR): a multicentre randomised controlled trial. Lancet 374 : $1351-1363$ 
10. Schmidt M, Stewart C, Bailey M, Nieszkowska A, Kelly J, Murphy L, Pilcher D, Cooper DJ, Scheinkestel C, Pellegrino V, Forrest P, Combes A, Hodgson C (2015) Mechanical ventilation management during extracorporeal membrane oxygenation for acute respiratory distress syndrome: a retrospective international multicenter study. Crit Care Med 43: 654-664

11. Marhong JD, Munshi L, Detsky M, Telesnicki T, Fan E (2015) Mechanical ventilation during extracorporeal life support (ECLS): a systematic review. Intensive Care Med 41: 994-1003

12. Terragni PP, Del Sorbo L, Mascia L, Urbino R, Martin EL, Birocco A, Faggiano C, Quintel M, Gattinoni L, Ranieri VM (2009) Tidal volume lower than $6 \mathrm{ml} / \mathrm{kg}$ enhances lung protection: role of extracorporeal carbon dioxide removal. Anesthesiology 111: 826-835

13. Bein T, Weber-Carstens S, Goldmann A, Müller T, Staudinger T, Brederlau J, Muellenbach R, Dembinski R, Graf BM, Wewalka M, Philipp A, Wernecke KD, Lubnow M, Slutsky AS (2013) Lower tidal volume strategy $(\approx 3 \mathrm{ml} / \mathrm{kg}$ ) combined with extracorporeal CO2 removal versus 'conventional' protective ventilation $(6 \mathrm{ml} / \mathrm{kg})$ in severe ARDS: the prospective randomized Xtravent-study. Intensive Care Med 39: 847-856.

\section{Figures}




\section{ECMO}

- Period: Nov 2014 - Sep 2019

- Cases identified: $N=103$

\section{Excluded:}

- cardiac failure resulting in venoarterial ECMO: $N=54$;

- ECMO provided for other indications than $\operatorname{ARF}(n=24)$;

- $\operatorname{pregnancy}(n=1)$;

- long-term chronic respiratory insufficiency treated with oxygen therapy or noninvasive ventilation $(n=1)$.

Patients included: $\boldsymbol{N}=\mathbf{2 3}$

including:

- 12 cases of viral pneumonia(One influenza

$B$, two influenza $A$ and nine avian influenza);

- 11 cases of non-viral infection.

Figure 1

Enrollment of patients. 
Basic characteristics

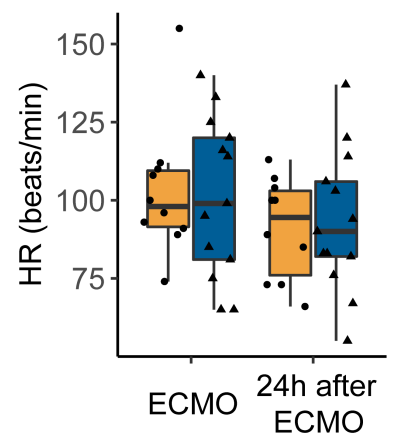

ECMO parameters

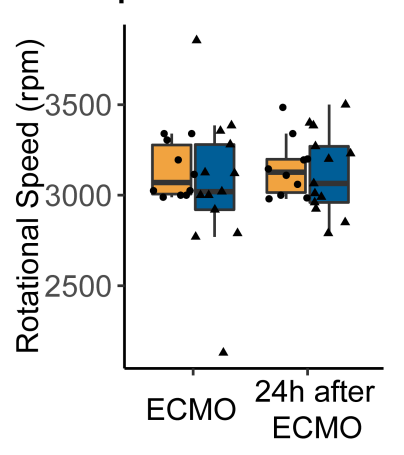

Blood Gas parameters
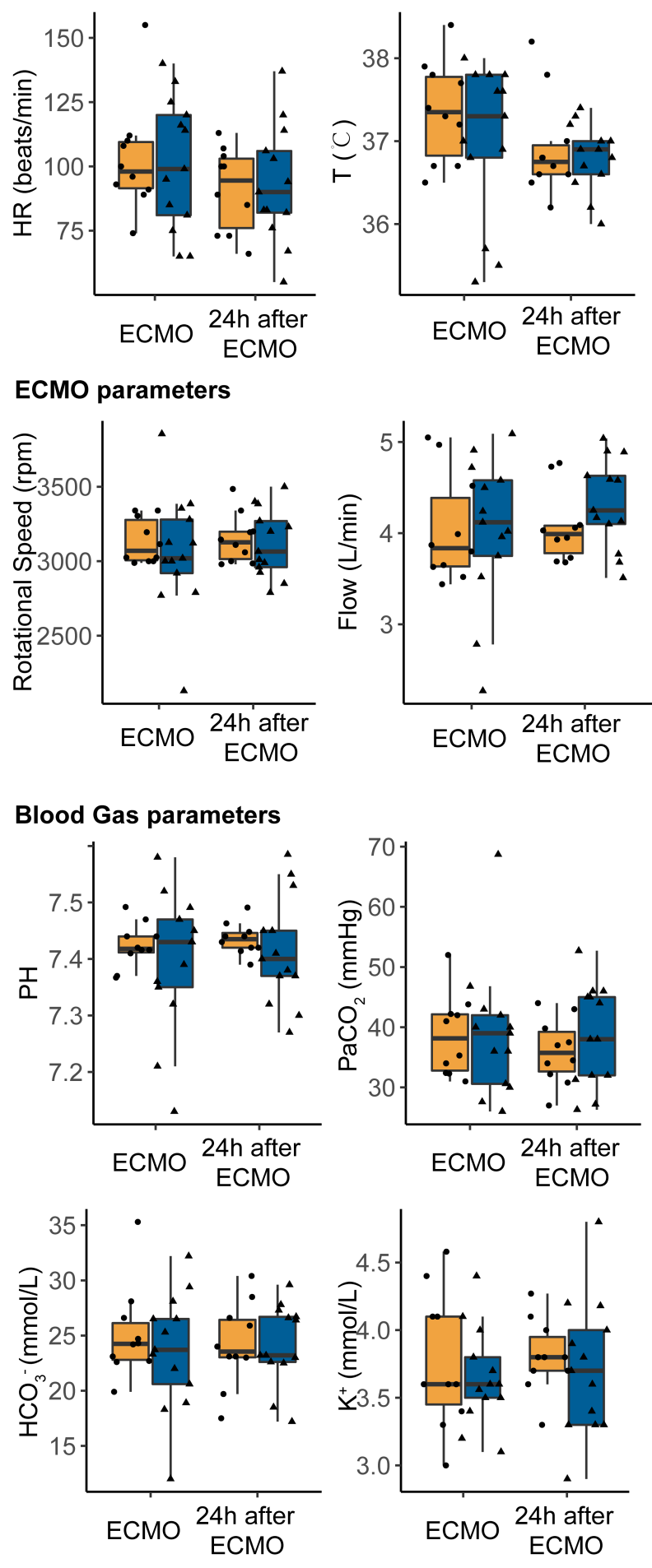

EG Group A

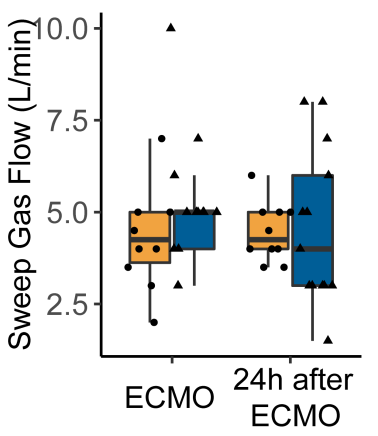

Group A Group B
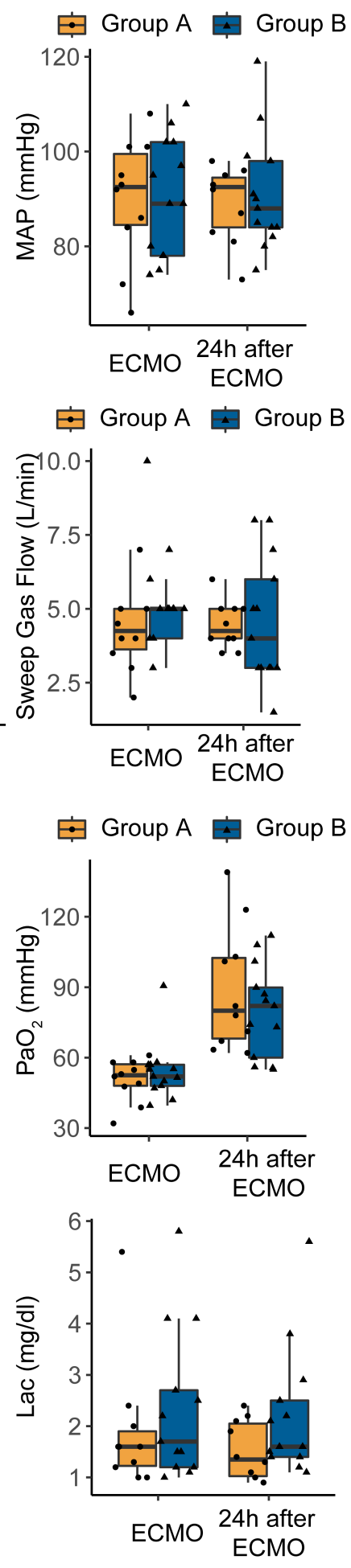

Figure 2

Basic characteristics, ECMO parameters and blood gas parameters of patients during ECMO and 24h after ECMO 


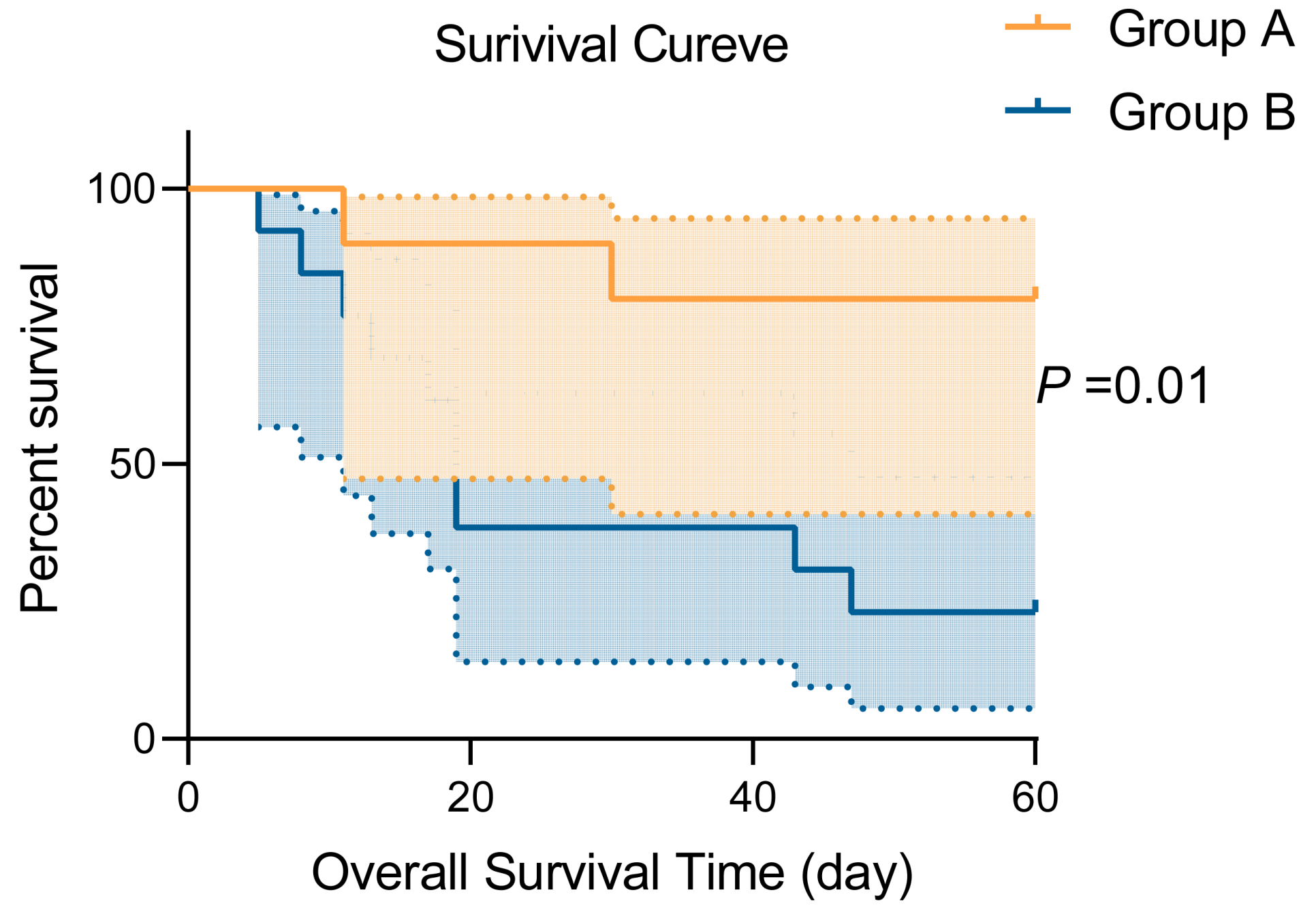

Figure 3

The survival curve of two groups. Red line represents the group A; Blue line represents the group B. 